\title{
Causes of childhood blindness in the People's Republic of China: results from 1131 blind school students in 18 provinces
}

\author{
S J Hornby, Y Xiao, C E Gilbert, A Foster, X Wang, X Liang, H Jing, L Wang, W Min, \\ Y Shi, Y Li
}

\begin{abstract}
International Centre for Eye Health, Department of Preventive Ophthalmology, Institute of Ophthalmology, London

S J Hornby

C E Gilbert

A Foster

The Red Cross Hospital of Yunnan, Qingnian Road, Kunming, PR China Y Xiao

$\mathrm{Y} \mathrm{Li}$

Inner Mongolia Medical University, No 1 Tong Dao North Street, Inner Mongolia, 010050 Huhhot, PR China

X Wang

Zhongshan

Ophthalmic Centre, 54 S Xianlie Road, Guangzhou 510060, PR China

$X$ Liang
\end{abstract}

China Medical University Shen Yang 110001, PR China H Jing

Xi Jing Eye Hospital, 4th Military Medical University Xian, Shaanxi 710032, PR China

L Wang

Sichuan Provincial People's Hospital, Chengdu, Sichuan 610072, PR China W Min

Xuzhou Eye Institute, 15 N Zhong-Shan Road, Xuzhou, Jiangsu 221002, PR China

Y Shi

Correspondence to: Miss Clare Gilbert, Institute of Ophthalmology, 11-43 Bath Street, London EC1V 9EL.

Accepted for publication 15 March 1999

\begin{abstract}
Aims-To determine the anatomical site and underlying causes of blindness and severe visual impairment in children under 16 years of age in special education in the People's Republic of China with a view to determining potentially preventable and treatable causes.

Methods-A national study of children attending schools for the blind in China was conducted between April and June 1998 using the WHO Prevention of Blindness Programme (WHO/PBL) eye examination record for children with blindness and low vision. Eight Chinese ophthalmologists attended a training workshop before conducting the study. 36 blind schools in 18 provinces of China were included.

Results-1245 children aged between 5 and 15 years were examined, of whom 1131 $(91 \%)$ were blind or severely visually impaired (visual acuity less than $6 / 60$ in the better eye). The commonest anatomical sites of visual loss were whole globe (mainly microphthalmos) $25.5 \%$ and retina (mainly dystrophies) $24.9 \%$. Lens was the major site in $\mathbf{1 8 . 8 \%}$, optic nerve in $13.6 \%$, and glaucoma in $9 \%$. Corneal scarring was not a major cause of visual loss. The aetiology was unknown in $52.9 \%$, hereditary factors were responsible in $30.7 \%$, and childhood causes in $14 \% .15 \%$ of cases were considered potentially preventable and $22.5 \%$ potentially treatable.

Conclusion-The pattern of childhood blindness seen in this study is likely to reflect the improved health and socioeconomic status of China but may partly reflect bias in admission to, and location of, blind schools, with higher socioeconomic groups overrepresented. Nutritional and infective causes of blindness are uncommon, and hereditary and unknown factors are now the predominant causes. (Br F Ophthalmol 1999;83:929-932)
\end{abstract}

For a child who is born blind or who becomes blind the total number of years of disability are greater than for a person who becomes blind later in life. Currently it is estimated that there are 1.5 million blind children in the world, of whom one million live in Asia. In order to set priorities for control programmes, baseline epidemiological data of the prevalence and major causes of childhood blindness are required. These are known to vary between regions and countries. ${ }^{1}$ No data are available for China.

The WHO Prevention of Blindness Programme with the International Centre for Eye Health has developed a standard methodology and reporting form to record the causes of visual loss in children with the emphasis on the identification of preventable or treatable causes of blindness. This methodology has now been used in many countries. ${ }^{2}$

China has a population of 1200 million (1996) of which 378 million are under 18 years of age. China's 22 provinces, five autonomous regions, and 5000 islands are governed from Beijing. (Hong Kong has a special status.) The population is mainly Han Chinese (over 90\%) but there are also 200 different ethnic minority groups. The country has a literacy rate of $82 \%$. Health indicators have shown a marked improvement in recent years - for example, the infant mortality (under 1 year) has improved from $140 / 1000$ live births in 1960 to $47 / 1000$ in 1996, and the under 5 mortality has improved from $209 / 1000$ to $47 / 1000$ over the same period. ${ }^{3}$

An epidemiological survey of blindness and low vision in China in 1987 examined 1579316 people. Data on low vision and blindness in children were reported together (that is, acuity of less than $6 / 18$ in the better eye). For children aged $0-13$ years the numerator can be calculated from the data given (390) but the denominator can only be estimated (415 000) giving a prevalence of visual impairment of approximately $0.94 / 1000 .{ }^{4}$ The leading causes in this study were hereditary factors in $48 \%$ followed by ametropia/amblyopia in $18 \%$, corneal disease in $9 \%$, and optic nerve disease in $7 \%$.

The aims of this study were:

- To identify the major anatomical site and underlying causes of blindness and severe visual impairment in children under 16 years of age in special education in the People's Republic of China

- To identify readily preventable or treatable causes - that is, the proportion of "avoidable blindness" in order to set priorities for control programmes.

- To provide baseline data to allow monitoring of changes in the pattern of causes of childhood blindness over time.

Materials and methods

Thirty eight schools for the blind were selected non-randomly for the study based on the 
Table 1 Categories of visual loss in 1245 Chinese children attending schools for the blind, 1998

\begin{tabular}{|c|c|c|c|}
\hline WHO category & $\begin{array}{l}\text { Level of } \\
\text { visual acuity } \\
\text { (better eye) }\end{array}$ & $n$ & $\%$ \\
\hline No impairment & $6 / 6-6 / 18$ & 22 & 1.8 \\
\hline Visual impairment & $<6 / 18-6 / 60$ & 92 & 7.4 \\
\hline Severe visual impairment & $<6 / 60-3 / 60$ & 156 & 12.5 \\
\hline Blind & $<3 / 60-\mathrm{NPL}$ & 970 & 77.9 \\
\hline $\begin{array}{l}\text { Cannot test } \\
\quad \text { (believed blind) }\end{array}$ & & 5 & 0.4 \\
\hline Total & & 1245 & 100 \\
\hline
\end{tabular}

Table 2 Anatomical site of abnormality leading to SVI/BL in 1131 children attending schools for the blind in China, 1998

\begin{tabular}{|c|c|c|c|c|c|c|}
\hline \multirow[b]{2}{*}{ Anatomical site } & \multicolumn{2}{|c|}{ Rural } & \multicolumn{2}{|c|}{ Urban } & \multicolumn{2}{|l|}{ Total } \\
\hline & $n$ & $\%$ & $n$ & $\%$ & $n$ & $\%$ \\
\hline Whole globe & 162 & 29.4 & 126 & 21.7 & 288 & 25.5 \\
\hline Cornea & 24 & 4.4 & 25 & 4.3 & 49 & 4.4 \\
\hline Lens & 87 & 15.8 & 126 & 21.7 & 213 & 18.8 \\
\hline Retina & 135 & 24.5 & 147 & 25.4 & 282 & 24.9 \\
\hline Uvea & 6 & 1.1 & 11 & 1.9 & 17 & 1.5 \\
\hline Optic nerve & 74 & 13.4 & 80 & 13.8 & 154 & 13.6 \\
\hline Glaucoma & 53 & 9.6 & 49 & 8.4 & 102 & 9.0 \\
\hline Others & 10 & 1.8 & 16 & 2.8 & 26 & 2.3 \\
\hline Total & 551 & 100 & 580 & 100 & 1131 & 100 \\
\hline
\end{tabular}

number of blind children in the school (30 or more children under 16), and to obtain as wide a geographic distribution as possible. Most of the schools were located in the capital cities of the provinces, with the exception of schools in Shandong Province. Each school was sent a written letter outlining the purpose of the study and requesting permission to visit the school and examine the children (two schools refused). Forms printed in English were used for the study and the equipment required was purchased in China. Eight Chinese ophthalmologists were identified to perform the study from provincial level hospitals in seven regions. They attended a training workshop in the standardised methodology before performing their part of the study in the different regions. The 36 schools in 18 provinces of mainland China were visited between April and June 1998.

All children under 16 present on the days of the visit were examined and each child was seen with his or her class teacher where possible. A brief history of the age of onset of visual loss, family history, history of consanguinity, and place of residence (village, town, or city) was taken by the examining ophthalmologist. Visual acuity was measured using an Illiterate Snellen E optotype with their current spectacles. Visual loss was classified according to the WHO categories of visual impairment (Table 1). ${ }^{5}$ Simple tests of functional vision were used, such as the ability to walk around chairs unaided, the ability to recognise faces, and to see printed shapes.

Anterior segment examination was performed with a torch and a magnifying loupe. The pupils were dilated with tropicamide $1 \%$ and the posterior segment was examined using a direct and indirect ophthalmoscope. Intraocular pressures were not measured.

All data were recorded on the WHO/PBL eye examination record form for children including the anatomical and aetiological classification. Data were entered into a database in EPI-INFO. Summary information in Chinese about each child's condition and any treatment required was given to the school. Those children requiring further assessment or treatment were referred to a local eye department and this was coordinated by the Amity Foundation, a Chinese non-governmental organisation involved in education of visually impaired children.

\section{Results}

In total, 1245 children between 5 and 15 years were examined, of whom 1131 were blind (BL) or severely visually impaired (SVI) (91\%). The results presented here relate to these children. Of those children $502(44.4 \%)$ had navigational vision-that is, were able to walk unaided around two chairs.

There were $745(65.9 \%)$ male and 386 $(34.1 \%)$ female children. The majority (83.6\%) were aged $13-15$ years: 551 children $(48.7 \%)$ came from a rural home and the remainder from urban homes $35.5 \%$ from a city and $15.8 \%$ from a town); $70 \%$ of children gave a history of visual loss since birth. There was a positive family history of another affected individual in $19.6 \%$. A history of consanguineous marriage of the parents was given in 56 cases $(4.8 \%$.) Consanguinity was more common among children from rural areas $(7.3 \%)$ than urban areas $(2.8 \%)$. The majority of children $(94 \%)$ had no other disability apart from blindness of the remainder the commonest associated disability was mental retardation in $4.3 \%$, deafness in $0.7 \%$ and other disabilities in $1 \%$.

ANATOMICAL CAUSES OF VISUAL LOSS

The anatomical classification of the causes of SVI/BL is shown in Table 2.

Congenital abnormalities of the globe were responsible for $16.6 \%$ of SVI/BL (15.3\% microphthalmos, $1.3 \%$ anophthalmos). Other abnormalities in this category (phthisis bulbi, disorganised eyes, or enucleated eyes) accounted for a further $8.9 \%$ of cases.

Corneal disease was seen in 49 children (4.4\%), caused by vitamin A deficiency in 19 . Disorders of the lens occurred in 213 children $(18.8 \%)$. Untreated cataract was responsible for visual loss in 69 children (6.1\%). Aphakia, usually with amblyopia, accounted for 138 children $(12.2 \%)$, and dislocated lenses in six $(0.5 \%)$.

Retinal causes were responsible for SVI/BL in $282(24.9 \%)$ children. The majority were retinal dystrophies $(179,15.8 \%)$ and albinism $(15,1.3 \%)$. Retinopathy of prematurity was diagnosed in 22 cases $(1.9 \%)$. Other retinal disorders, including retinal detachment and retinoblastoma, accounted for the remainder $(5.9 \%)$.

Uveal disorders were identified in $1.5 \%$ of children (aniridia (nine), coloboma (one), uveitis (seven)), and optic nerve disorders were seen in $13.6 \%$ of children (optic atrophy in 87 $(7.7 \%)$ and optic nerve hypoplasia in 67 $(5.9 \%))$. Buphthalmos and glaucoma were diagnosed in 102 cases $(9.0 \%)$. Other causes 
Table 3 Aetiological classification in 1131 children with SVI/BL attending schools for the blind in China, 1998

\begin{tabular}{|c|c|c|c|c|c|c|}
\hline \multirow[b]{2}{*}{ Aetiology } & \multicolumn{2}{|c|}{ Rural } & \multicolumn{2}{|c|}{ Urban } & \multicolumn{2}{|l|}{ Total } \\
\hline & n & $\%$ & $n$ & $\%$ & $n$ & $\%$ \\
\hline Hereditary & 163 & 29.6 & 184 & 31.7 & 347 & 30.7 \\
\hline Intrauterine & 0 & 0 & 1 & 0.2 & 1 & 0.1 \\
\hline Perinatal & 8 & 1.5 & 18 & 3.1 & 26 & 2.3 \\
\hline Childhood & 85 & 15.4 & 73 & 13.6 & 158 & 14.0 \\
\hline Unknown & 295 & 53.5 & 304 & 52.4 & 599 & 52.9 \\
\hline Total & 551 & 100 & 580 & 100 & 1131 & 100 \\
\hline
\end{tabular}

Table 4 Avoidable causes of SVI/BL in 1131 children attending schools for the blind in China, 1998

\begin{tabular}{|c|c|c|c|c|c|c|}
\hline \multirow{2}{*}{$\begin{array}{l}\text { Avoidable } \\
\text { causes }\end{array}$} & \multicolumn{2}{|c|}{$\begin{array}{l}\text { Rural } \\
(n=550)\end{array}$} & \multicolumn{2}{|c|}{$\begin{array}{l}\text { Urban } \\
(n=580)\end{array}$} & \multicolumn{2}{|l|}{ Total } \\
\hline & $n$ & $\%$ & $n$ & $\%$ & $n$ & $\%$ \\
\hline Preventable & 73 & 13.2 & 96 & 16.5 & 169 & 15.0 \\
\hline $\begin{array}{l}\text { Autosomal } \\
\text { dominant }\end{array}$ & 27 & 4 & 60 & 10.3 & 87 & 7.7 \\
\hline $\mathrm{VAD} /$ measles & 14 & 2.5 & 16 & 2.8 & 30 & 2.7 \\
\hline Trauma & 32 & 5.8 & 20 & 3.4 & 52 & 4.6 \\
\hline Treatable & 116 & 21.0 & 139 & 23.9 & 255 & 22.5 \\
\hline Cataract & 58 & 10.5 & 75 & 12.9 & 133 & 11.8 \\
\hline Glaucoma & 46 & 8.3 & 47 & 8.1 & 93 & 8.2 \\
\hline ROP & 7 & 1.3 & 15 & 2.6 & 22 & 1.9 \\
\hline Uveitis & 5 & 0.9 & 2 & 0.3 & 7 & 0.6 \\
\hline Total & 189 & 34.2 & 235 & 40.4 & 424 & 37.5 \\
\hline
\end{tabular}

where the globe appeared normal accounted for the remaining 26 cases $(2.3 \%)$. The disorders included high refractive errors and amblyopia in $13(1.1 \%)$, idiopathic nystagmus in eight, and cortical blindness in four.

AETIOLOGY OF VISUAL LOSS

The aetiology of SVI/BL, classified according to the time of known insult resulting in visual loss, is shown in Table 3. The largest aetiological category was the unknown group $(52.9 \%)$ followed by hereditary factors in $30.7 \%$, childhood factors in $14 \%$, perinatal factors in $2.3 \%$, and intrauterine factors in $0.1 \%$. In the unknown aetiology group, 218 of 599 children had congenital anomalies of prenatal origin, which could be due to genetic or intrauterine factors. When these children are included with those with identified hereditary and intrauterine factors, $50 \%$ of cases of SVI/BL are attributable to prenatal factors. The aetiological classifications for rural and urban children are compared in Table 3, showing little difference between the two groups.

AVOIDABLE CAUSES

Overall, $37.5 \%$ of children had potentially "avoidable" causes of blindness - that is, preventable causes $(15 \%)$ and treatable causes $(22.5 \%)$, of which cataract was the first most important cause and glaucoma the second (Table 4).

\section{Discussion}

Studies such as this provide information on the causes of blindness in children in schools for the blind but the findings have to be interpreted with caution as the data are not population based. The advantages are that a large number of children with visual loss can be examined in a short time, relatively inexpensively, and the information does provide an indication of the relative importance of the dif- ferent causes of childhood blindness. However, they are subject to certain inherent biases. For example, children with multiple disabilities, preschool age children, those who have died, those from lower socioeconomic groups, or from rural communities are likely to be underrepresented. These biases may be more marked in China than in some other countries studied previously as there are no schools for the blind in the sparsely populated, poorer western part of China. All the schools were residential. Many of the schools reported difficulty in recruiting younger children. The cost of schooling the children was given as a major reason for failing to attend or dropping out of school. In most countries the prevalence of childhood blindness is higher in poorer regions but the majority of blind schools in China are in the relatively affluent eastern coastal area. Causes of blindness associated with poverty and a high mortality such as vitamin A deficiency are likely therefore to be underrepresented in this study.

The results of this study show a mixed picture of causes with a predominance of hereditary and unknown aetiologies, a pattern similar to that in industrialised countries. The pattern of childhood blindness is very different from that seen in the developing countries of Africa, where corneal scarring from vitamin A deficiency, measles, and harmful traditional eye medicines predominate. ${ }^{6}$ In India also the major cause of SVI/BL in children in blind schools is vitamin A deficiency. ${ }^{7}$ In this study only $2.7 \%$ of SVI/BL was attributed to vitamin A deficiency.

Overall, $30.7 \%$ of SVI/BL in China was attributed to genetic disease, most commonly retinal dystrophies. The mode of inheritance was autosomal dominant in $7.7 \%$, recessive in $5.8 \%$, $\mathrm{X}$ linked in $0.3 \%$, and undetermined in $16.9 \%$. Given the small family sizes in China it is likely that hereditary diseases would be underdiagnosed, as siblings are uncommon because of the "one child policy". Consanguinity was uncommon (4.8\%) and is generally declining, possibly as a result of increasing urbanisation and education. Before the second world war first cousin marriage (termed "up the hill") was quite common among the Han Chinese and also among certain minority Muslim groups. ${ }^{8}$

In industrialised nations hereditary disease accounts for up to half of cases of childhood blindness. In comparison, studies using the same methodology in other countries of Asia found genetic disease to be responsible for $23.0 \%$ of SVI/BL in India $735.0 \%$ in Sri Lanka, ${ }^{9}$ and $16.8 \%$ in Thailand and the Philippines. ${ }^{10}$ In contrast with China countries with high levels of consanguineous marriage such as India and Sri Lanka had a high proportion of autosomal recessive disease. ${ }^{11}$

Nineteen per cent of children had SVI/BL from disorders of the lens. Of these $65 \%$ were aphakic and/or amblyopic probably as a result of late surgery or inadequate refractive correction with $32 \%$ having unoperated cataracts.

Congenital ocular anomalies (microphthalmos, anophthalmos, and coloboma) accounted 
for $16.7 \%$ of children with SVI/BL. Of these $15.8 \%$ were attributed to hereditary factors but the remainder were due to unknown prenatal factors. The proportion of SVI/BL due to congenital anomalies is higher than in most other countries studied but less than in Sri Lanka and in India. Known causes of congenital eye anomalies are genetic disease, both monogenetic and chromosomal, and certain teratogens such as maternal infections or drugs such as alcohol. However, the majority of cases are of unknown aetiology. ${ }^{12}$

Optic nerve disease (atrophy and hypoplasia) was responsible for $13.6 \%$ of SVI/BL which is higher than in India $(5.9 \%) .^{7}$ The majority of optic atrophy was attributed to childhood factors such as trauma, tumours, or meningitis $(68 \%)$. Optic atrophy accounts for more than $25 \%$ of childhood blindness in England. ${ }^{13}$ Optic nerve hypoplasia is known to be a heterogeneous condition and is usually of unknown aetiology (prenatal factors).

Glaucoma or buphthalmos was responsible for $9 \%$ of BL/SVI. This is similar to studies in west Africa and Latin America but more than in other countries studied in Asia. ${ }^{1}$

Retinopathy of prematurity (ROP) was seen in $2 \%$ of children with SVI/BL. There were twice as many cases with ROP in the urban area compared with the rural area but the numbers were small. Neonatal intensive care facilities are now becoming available in China although facilities for monitoring oxygen levels are limited. It is likely that ROP may increase in China as in other industrialising countries of Asia similar to what has occurred in Latin America where ROP is a major cause of blindness in children. There is at least a 5 year time lag (age of school entry) before a newly emerging blinding condition will be reflected in a blind school study. ${ }^{14}$ The majority of children in this study were aged over 12 years.

Overall, $15 \%$ of SVI/BL in this study was potentially preventable. This included autosomal dominant disease in $7.7 \%$ (which may be preventable by genetic counselling), trauma in $4.6 \%$, and vitamin $\mathrm{A}$ deficiency/measles in $2.7 \%$.

Causes which are potentially treatable were responsible for $22.5 \%$. These include cataract, glaucoma, ROP, and uveitis. These findings suggest that there is a need for specialist paediatric ophthalmic services for surgical management or these conditions and optical management of associated refractive error and amblyopia. There is also a need for screening for early detection of cataract, as early referral and management would improve the prognosis from surgery. The provincial level hospitals in China are generally well equipped but access to these facilities is not always straightforward and treatment may be expensive.

The schools for the blind in China do not generally accept students with multiple handicaps except if the additional handicaps are mild; $94 \%$ of the children had no disability apart from blindness. This is in contrast with schools for the blind in the UK and USA where a high proportion of children have additional disabilities often associated with cortical visual problems.

\section{Conclusions}

The pattern of childhood blindness seen in this study almost certainly reflects the improved primary healthcare and socioeconomic status of China, reflected in dramatic reductions in under 5 mortality rates over the past 30 years. Nutritional and infective causes of blindness are uncommon and hereditary and unknown factors are now predominant causes, as in Western countries. This may partly reflect bias in admission to blind schools, which places a significant economic burden on parents and so is likely to reflect the pattern of blindness among the higher socioeconomic groups. However, there was little difference in causes in children from rural areas, who are likely to be poorer compared with those from urban homes. There is a need for specialist paediatric ophthalmic services to treat paediatric cataract and glaucoma. Retinopathy of prematurity is not a major cause of SVI/BL at present in school age children but may increase in the future, and so surveillance for this condition is important.

This study was supported by grants from the Asian Foundation for the Prevention of Blindness and Christoffel Blindenmission. Miss She Hongyu of the Amity Foundation was invaluable in the organisation, planning, and logistics of this survey. $\mathrm{Dr}$ Michael Li identified the Chinese ophthalmologists who took part. The Nanjing School for the Blind hosted the training workshop.

1 Foster A, Gilbert C. Epidemiology of childhood blindness. Eye 1992;6:173-6.

2 Gilbert C, Foster A, Negrel AD, et al. Childhood blindness: a new form for recording causes of visual loss in children. Bull World Health Organ 1993;71:485-9.

3 UNICEF. The state of the world's children. New York: Oxford University Press, 1998

4 Shi-yuan Z, Liu-he Z, Yong-qing G. Epidemiological survey of blindness and low vision in China. Chinese Med $\mathcal{F}$ 1992; 105:603-8.

5 World Health Organisation. Adaptation of the international classification of diseases. 1975 revision. Geneva: WHO, 1977.

6 Foster A, Sommer A. Corneal ulceration, measles, and childhood blindness in Tanzania. Br f Ophthalmol 1987;71: 331-43.

7 Rahi JS, Sripathi S, Gilbert CE, et al. Childhood blindness in India: causes in 1318 blind school students in nine in India: causes in 1318

8 Bittles AH, Mason W, Greene J, et al. Reproductive behavious and health in consanguineous marriages. Science 1991; 252:789-94.

9 Eckstein MB, Foster A, Gilbert CE. Causes of childhood blindness in Sri Lanka: results from children attending six schools for the blind. Br F Ophthalmol 1995;79:633-6.

10 Gilbert C, Foster A. Causes of blindness in children attending four schools for the blind in Thailand and the Philippines. Int Ophthalmol 1993;17:229-34.

11 Gilbert C, Rahi J, Eckstein M, et al. Hereditary disease as a cause of childhood blindness: regional variation. Results of blind school studies undertaken in countries of Latin America, Asia and Africa. Ophthalmic Genet 1995;16:1-10. boma. F Med Genet 1993;30:664-9.

Department of Health. Statistical bulletin. 5/86. London: DHSS, 1998

14 Gilbert C, Rahi J, Eckstein M, et al. Retinopathy of prematurity in middle-income countries. Lancet 1997;350:12-14. 